The researchers have also demonstrated that graphene can serve as a supercurrent transistor. Heersche and colleagues have created a device in which the supercurrent can be regulated by a voltage, similar to the control exhibited in a conventional semiconductor transistor in which the current is controlled by a gate electrode. Graphene is not a semiconductor, but rather a semi-metal. This means that not only the size of the supercurrent can be regulated, but also the type of charge carrier, according to the researchers. This can be electron Cooper pairs (with a negative charge) or hole Cooper pairs (with a positive charge). The researchers therefore report the fabrication of a bipolar supercurrent transistor.

The researchers said that, in sweeping the position of the Fermi level from the valence band to the conduction band, they sweep through the Dirac point. They said that, even then, the supercurrent remains finite, which they argue demonstrates that the electronic transport in graphene is phase coherent, even when the Fermi level is located at the Dirac point.

The researchers also discuss the effects of time reversal symmetry in graphene in the superconducting state based upon occupation of the two different K-points in the band structure by the two Cooper electrons. They contrast this to graphene in the normal state in which transport is determined by a single-valley band structure.

\section{Photoconductive Atomic Force Microscopy Maps Photocurrent in Solar Cells}

Solar cells made from organic semiconductor blends are currently low in power conversion efficiency. Typically, bound excitons created from absorbed light must dissociate into free charges at donoracceptor interfaces. However, efficient light absorption requires a $\sim 100$-nm film thickness, but excitons can only travel $\sim 10 \mathrm{~nm}$ before they decay. This so-called exciton bottleneck can be overcome with nanostructured organic solar cells with large internal surface areas. For example, donor and acceptor materials can be processed in a common solvent to a phase-separated film. Solar cell performance has been shown to depend very much on film morphology, but conventional characterization tools either lack the resolution needed to approach the exciton diffusion length, or do not provide direct information on the correlation between structure and local per- formance. Recently, however, assistant professors D.S. Ginger and G.P. Bartholomew, along with their students D. Coffey, O. Reid, and D. Rodovsky at the Department of Chemistry, University of Washington, Seattle, have demonstrated that photoconductive atomic force microscopy (pcAFM) can map local photocurrents in polymer-fullerene blend solar cells with 20-nm resolution.

As reported in the March issue of Nano Letters (p. 738; DOI: 10.1021/nl062989e), Ginger and co-researchers applied pcAFM to poly[2-methoxy-5-(3',7'dimethyloctyl-oxy)-1,4-phenylene vinylene]:(6,6)-phenyl- $\mathrm{C}_{61}$-butyric acid methyl ester (MDMO-PPV:PCBM) — a well known photovoltaic blend. Standard, mm-sized photovoltaic cells were fabricated by spincoating and curing a 40-nm layer of PEDOT:PSS (a conductive mixture of two ionic polymers) onto glass substrates coated with indium tin oxide (ITO). An 80-nm thick film of MDMO-PPV:PCBM was then spin-coated onto the ITO from either chlorobenzene, xylene, or toluene solutions in order to obtain varying degrees of phase separation in the active layer. Aluminum top contacts 40 -nm thick were evaporated through a shadow mask, thereby defining
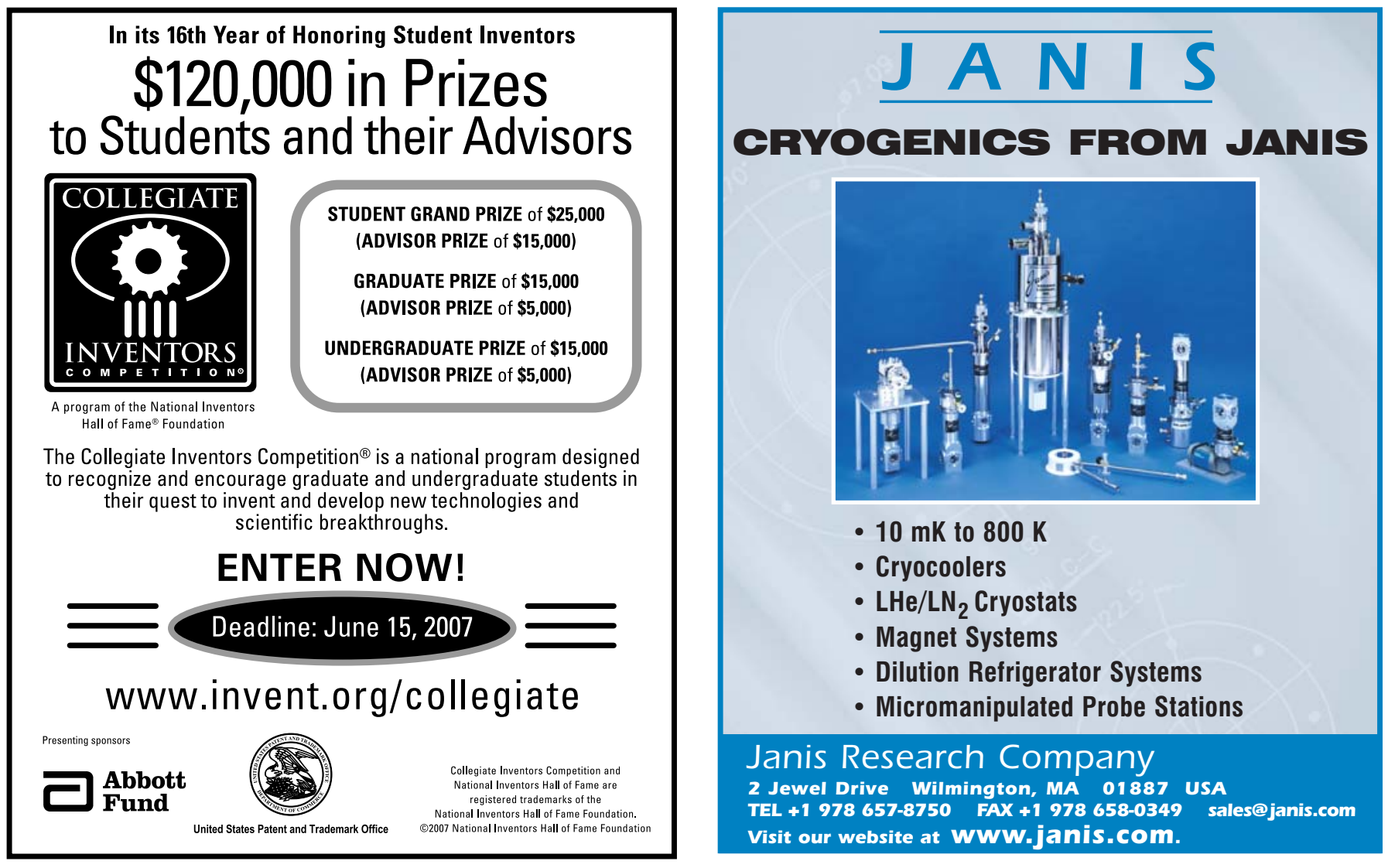


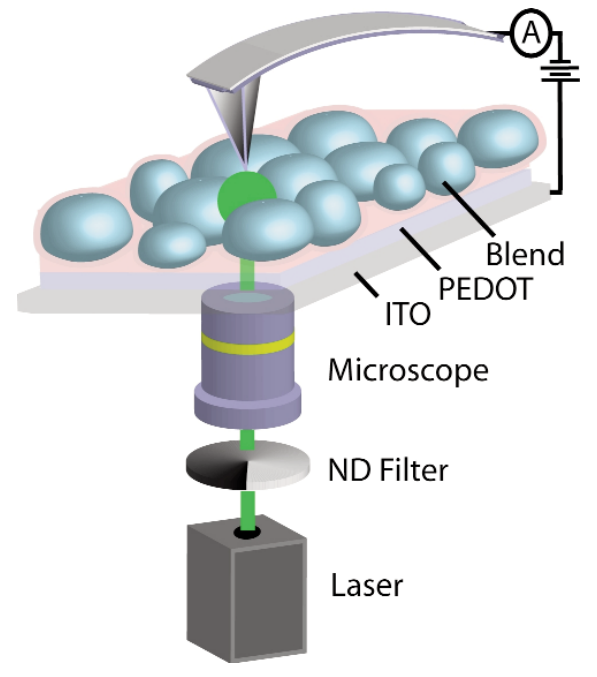

Figure 1. Schematic of a laser focused through a transparent electrode onto a photovoltaic blend film. Current is collected with a metal-coated atomic force microscope tip.

active devices with an area of $1.5 \mathrm{~mm}^{2}$ (see Figure 1).

In between the $\mathrm{Al}$ electrodes of a device, the researchers aligned a conductive AFM probe at the center of a diffraction-limited laser $(532 \mathrm{~nm})$ spot, which generated a photocurrent signal sufficient for imaging at current levels in the range of $\sim 1-10 \mathrm{pA}$ at low intensity or at $\sim 1 \mathrm{nA}$ at high intensity, corresponding to observation of the photocurrent intensity dependence over nearly six orders of magnitude in illumination intensity.

The research team collected photocurrent images under short circuit conditions (zero applied bias) as well as with various voltages applied between the ITO layer and the conductive, Pt-coated AFM probe tip. Contact mode was used to simultaneously measure the current and film height. The researchers observed variations in photocurrent by as much as a factor of three between invididual PCBM domains with similar topography. They attributed these variations to differences in vertical film structure. The researchers speculate that "MDMO-PPV-rich regions generate low photocurrents because of poor charge mobility and/or energy level alignment" whereas "PCBM-rich regions generate more photocurrent, but the vertical position of a PCBM-rich domain and domain-stacking can limit electron extraction or enhance electron extraction depending on the thickness of the MDMO-PPV:PCBM overlayer."

In addition, the researchers observed smaller photocurrent fluctuations on a length scale in the range of $200-600 \mathrm{~nm}$. Photocurrents averaged from hundreds of measurements made with the Pt tip agree well with the photocurrents measured for bulk devices with Al contacts, demonstrating that the pcAFM measurements provide information relevant to device operation, despite the differences in geometry and intensity.

The researchers concluded that local heterogeneity diminishes solar cell performance; gaining greater control and regularity of film morphology in both lateral and vertical directions will improve performance. In addition, the researchers said that their technique's "simplicity should allow for the characterization of many other organic blends over a wide range of illumination intensity. Further, [because] this technique is based on standard current-voltage measurements, albeit on solar cells the size of the AFM tip, it is interesting to speculate that other current-voltage based characterizations, including spacecharge limited photocurrent and time-offlight mobility measurements, might be accessible with high resolution."

STEVEN TROHALAKI

\section{Temperature Limit Set for Future Superconducting Magnet}

The material currently used in magnetic resonance (MR) imaging machines in both hospitals and research laboratories-a low-temperature superconducting alloy of the metallic element niobium-has been pushed almost as far as it can go in achieving a magnetic field, of 21 Tesla. "A holy grail of the scientific community, as set out recently by the National Research Council, is to build a superconducting magnet of 30 Tesla," said William Halperin of Northwestern University. Now, a high-temperature superconductor-Bi-2212, a compound containing bismuth-has been identified as a material that might be suitable for the new wires needed to one day build a 30-Tesla magnet. A report on this finding has been published in the April issue of Nature Physics (p. 239; DOI: $10.1038 /$ nphys540) by Halperin and B. Chen of Northwestern, P. Guptasarma of the University of Wisconsin-Milwaukee, D.G. Hinks of Argonne National Laboratory, V.F. Mitrovic of Brown University, and A.P. Reyes and P.L. Kuhns of the National High Magnetic Field Laboratory in Tallahassee, Fla.

Halperin said, "In MR imaging, the higher the magnetic field, the higher the resolution, which provides scientists with more detail for analysis. A 30-Tesla magnet could drive significant advances in chemistry, biology, and medicine."

A superconductor, when cooled to its appropriate temperature, conducts electricity without any resistance. Superconductivity first appears in Bi-2212 at a high temperature of $90 \mathrm{~K}$, but Halperin and his colleagues found that the stable state required in high-magnetic fields can be established only when the temperature falls below $12 \mathrm{~K}$. The team established this limit for Bi-2212.

Using MR techniques at the National High Magnetic Field Laboratory, Halperin and his team studied Bi-2212. To measure its properties, they put the rare isotope oxygen-17 into a crystal of Bi-2212, with the isotope acting as a probe, much like a fluorescent dye. They then determined the phase diagram of the material where superconductivity is stable, which showed high temperature and high magnetic field could not be achieved together.

"To create a 30-Tesla magnet, we need a superconducting material that can carry the required amount of electricity without blowing up," said Halperin; "We have found that the operating temperature for Bi-2212 must be below $12 \mathrm{~K}$. The good news is that this temperature can be reached by cooling the magnet with liquid helium. If we had found the upper limit to be $2 \mathrm{~K}$ then the cryogenic requirements would be intractable."

"Sometimes what seems to be bad can be good," said Chen, lead author of the article and a graduate student of Halperin's. "Our findings set a speed limit. If you go beyond this speed you may have trouble. Knowing the upper temperature limit is a kind of security."

Halperin said, "Now that we have this information about Bi-2212, the next question is, 'Can such a magnet actually be made?'”

\section{Flow of Tiny Bubbles Mimics Computer Circuitry}

In work that could dramatically boost the capabilities of "lab-on-a-chip" devices, researchers at the Massachusetts Institute of Technology have created a way to use tiny bubbles to mimic the capabilities of a computer. The team, based at MIT's Center for Bits and Atoms, reports in the February 9 issue of Science (p. 832; DOI: 10.1126 /science.1136907) that the bubbles in their microfluidic device can carry onchip process control information, just like the electronic circuits of a traditional microprocessor, while also performing chemical reactions.

Researcher N. Gershenfeld, director of the Center for Bits and Atoms, said, "Bubble logic merges chemistry with computation, allowing a digital bit to carry a chemical payload. Until now, there was a clear distinction between the 